\title{
NAFLD in the Elderly
}

\author{
Saleh A Alqahtani $\mathbb{D}^{1,2}$ \\ Jörn M Schattenberg $\left(\mathbb{D}^{3}\right.$
}

'Liver Transplantation Center, King Faisal Specialist Hospital \& Research Center, Riyadh, Saudi Arabia; ${ }^{2}$ Division of Gastroenterology and Hepatology, Johns Hopkins University, Baltimore, MD, USA; ${ }^{3}$ Metabolic Liver Research Program, I. Department of Medicine, University Medical Center, Mainz, Germany
Correspondence: Jörn M Schattenberg Metabolic Liver Research Program, I. Department of Medicine, University Medical Center of the Johannes GutenbergUniversity, Langenbeckstrasse I, Mainz, 55I3I, Germany Email joern.schattenberg@unimedizinmainz.de

\begin{abstract}
Non-alcoholic fatty liver disease (NAFLD) is an increasingly prevalent disease globally. Current estimates are that $24 \%$ of the adult population, thus, one billion individuals worldwide, are affected. Interestingly, the prevalence of fatty liver seems to peak between 40-50 years of age in males and 60-69 years in females, often slightly decreasing in older (>70 years) cohorts. Furthermore, several risk factors for NAFLD development, such as hypertension, diabetes, hyperlipidemia, and obesity are higher in older adults. The diagnosis and management strategies in older adults are sometimes challenging, and certain agespecific factors have to be taken into account by healthcare professionals. In this review, we provide an overview of considerations relevant to the management and diagnosis of NAFLD in older adults (age $>65$ years) and discuss the types of pharmacological interventions available for the management of non-alcoholic steatohepatitis (NASH) in the aging population.
\end{abstract}

Keywords: liver disease, aging, fatty liver, NASH, senescence, regeneration

\section{The Aged Liver}

Aging is accompanied by progressive physiological alterations, which gradually disturb homeostasis and results in functional decline and frailty. ${ }^{1}$ Older adults are more susceptible to several non-communicable diseases, such as cancer, cardiovascular disease, hypertension, diabetes, chronic obstructive pulmonary disease, cerebrovascular disease, hearing loss, dementia, arthritis, and several others. ${ }^{2}$ All of these complications can, in part, be explained at a cellular level by the "hallmarks of aging." These hallmarks encompass several cellular processes that become less efficient or dysregulated with age, such as genomic instability, telomere attrition, impaired proteostasis, epigenetic alterations, impaired nutrient-sensing capacity, mitochondrial dysfunction, senescence, stem cell exhaustion, and altered intercellular communication (see Figure 1). ${ }^{3}$

\section{Telomere Shortening and Impaired Proteostasis}

The liver has a remarkable capacity for self-regeneration. In the healthy liver, regeneration, and repair are driven by mitogenic growth factors and cytokines. However, these age-mediated changes impair the regenerative capacity of the liver. For example, cells from liver tissue of older patients have shorter telomeres, which leads to irreversible cellular growth arrest and compromised proliferation. ${ }^{4}$ Impaired autophagy is also of relevance in the aged liver, as autophagy is necessary for the turnover of misfolded proteins and organelles, such as mitochondria in both homeostatic as well as pathological conditions and the mobilization of lipid reserves during starvation. ${ }^{5}$ Lessons from animal models show that aged mouse 

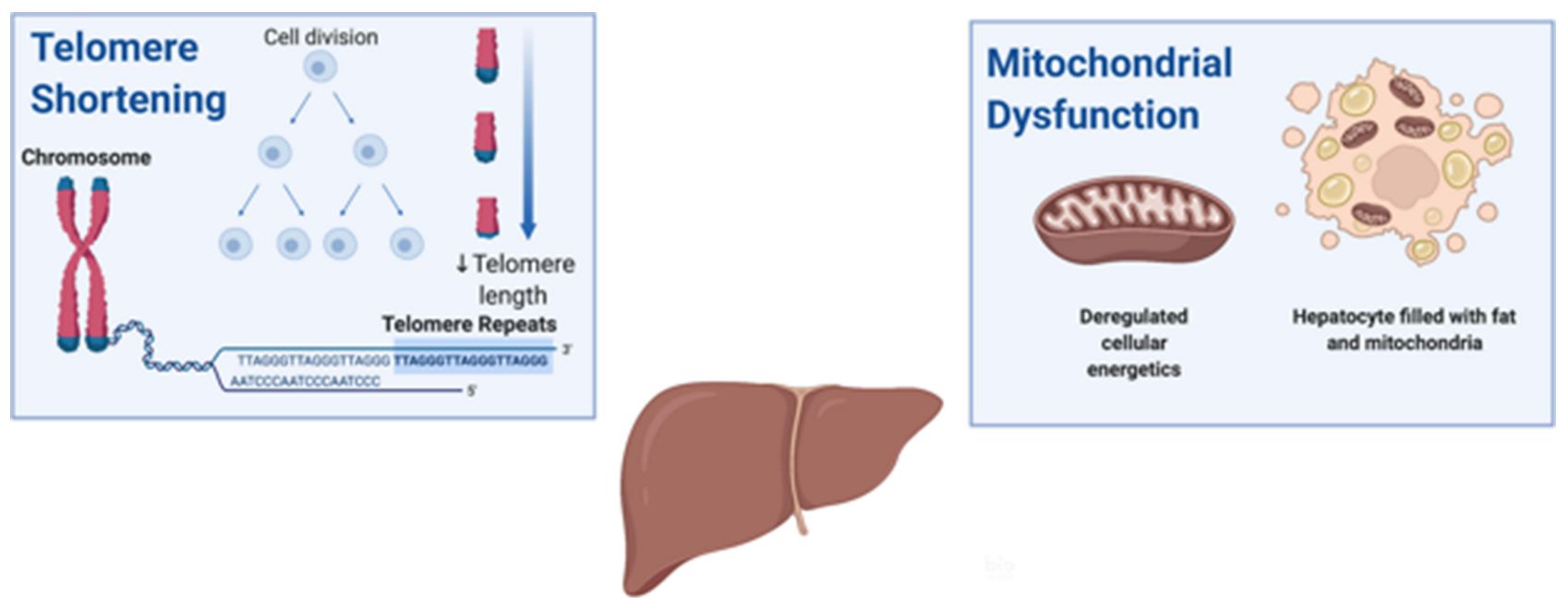

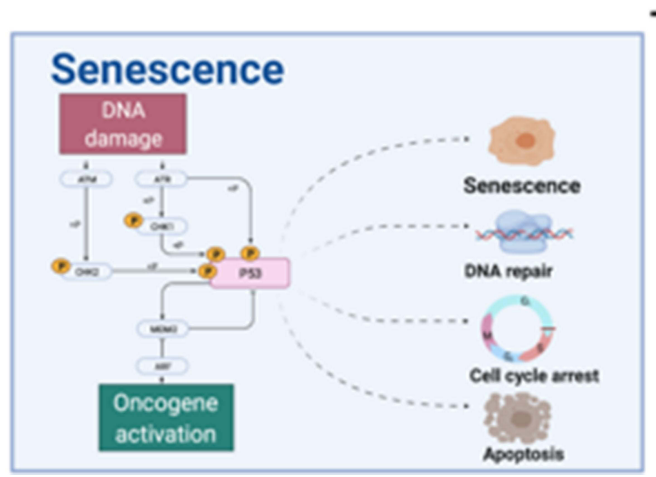

The aging liver

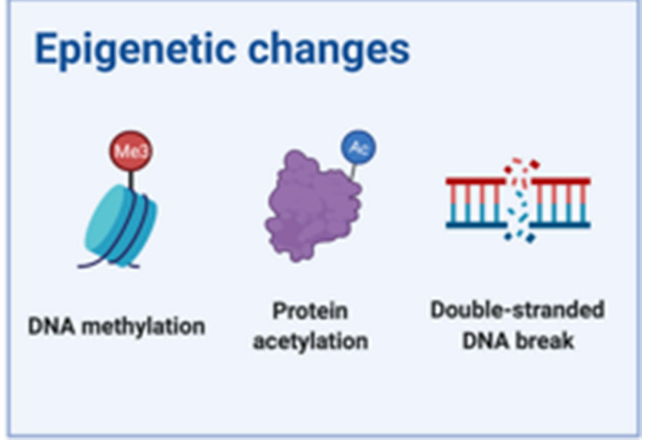

Figure I Mechanisms of NAFLD and the aging liver. Created with BioRender.com

and rat hepatocytes present a higher content of misfolded protein aggregates as a result of defective autophagy. ${ }^{6}$ These aggregates are known to promote the production of reactive oxygen species (ROS) that further negatively impact cellular autophagy, leading to a potential negative feedback loop. ${ }^{7}$ Data on humans are scarce, thus, an avenue to explore would be to determine whether autophagyinducing drugs could delay or reverse autophagy-mediated liver defects.

\section{Cellular Senescence}

Senescence, on a cellular level, is broadly defined as a state of irreversible cell cycle arrest that can arise as a consequence of different damage, such as telomere attrition, oxidative stress, and DNA damage. ${ }^{8,9}$ Once cells turn senescent, they become desensitized to mitogenic stimuli and are less prone to undergo apoptosis. ${ }^{10}$ Several studies propose that cycles of liver damage and repair exhaust telomeres in existing cells, accelerate the process of senescence and, consequently, triggers cirrhosis. ${ }^{11}$ Furthermore, a specific polymorphism in the CDKN1A gene has been associated with progression to fibrosis in NAFLD patients. ${ }^{12}$ Adverse outcomes of liver diseases have been previously associated with higher amounts of p21 expression and hepatocyte senescence. ${ }^{13,14}$ Therefore, senescence may be a mediator of NAFLD progression and could potentially be targeted therapeutically to prevent or delay the development of liver fibrosis. Interestingly, the natural aging process per se does not seem to cause senescence in either cholangiocytes or hepatocytes: these cells display no telomere shortening caused by aging, as the reported telomere shortening is exclusive to Kupffer and stellate cells. ${ }^{15}$ Thus, it is unknown why telomere shortening only occurs in a subset of cells. Similar results have been reported in mice and human hepatocytes, which can undergo extensive proliferation in vivo and escape senescence for at least twelve rounds of proliferation. ${ }^{16}$ In mice, depletion of senescent cells through a gene-mediated strategy or by administration of two senolytic drugs (dasatinib and quercetin) reduced hepatic steatosis. At the same time, mice with a genetic mutation that favors the induction of hepatic senescence tend to accumulate more fat in the liver. ${ }^{17}$ Taken together, all these data suggest that liver-specific senescence precedes or predisposes to the development of a fatty liver. Therefore, 
a healthy lifestyle may be sufficient to prevent or delay senescence, and potentially, NAFLD. In the future, senolytic drugs may be employed to prevent the progression of NAFLD.

\section{Epigenetic Alterations}

Regulation of CDKN1A expression, not only across different cells but also throughout time in the same cell, is a requirement for the development and maintenance of human life. Briefly, epigenetics encompasses both the establishment and maintenance of specific transcriptional networks without associated changes to the underlying sequence of DNA. The dysregulation of epigenetic mechanisms across different tissues occurs with aging, contributing to age-related diseases. ${ }^{18}$ In the case of liver disease, it has been estimated that $\sim 50 \%$ of all hepatocellular carcinomas (HCCs) involve mutations in genes encoding proteins that act as chromatin regulators. Epigenetic modifications have already been associated with NAFLD development and proposed as a link between lifestyle and NAFLD development. For example, in mice, a methyl-deficient diet leads to generalized DNA demethylation in the liver, along with a lower expression of a protein involved in the DNA methylation process: DNA methyltransferase 1 (DNMT1). Further, the level of trimethylation of histones H3K27 and H4K20 is also reduced. Altogether, these patterns were proposed to compromise genomic integrity through the modulation of heterochromatin reorganization. ${ }^{19}$ DNMT1 has been reported to carry out the methylation of mitochondrial DNA, specifically of the cytosine at the carbon-5 position of $\mathrm{CpG}$ dinucleotides, regulating the expression of mitochondrial genes. ${ }^{20}$ In humans, methylation of the mitochondrial ND6 gene was higher in patients in NASH when compared with those with simple steatosis, and the degree of ND6 methylation was associated with the NAFLD activity score. These results were potentially mediated by a higher expression of DNMT1 in the liver of patients with NASH. ${ }^{21}$ Interestingly, the aged liver appears to have a distinct epigenetic signature, with differential methylation in regions associated with the WNT pathway and epithelial-to-mesenchymal transition. ${ }^{22}$ If and how these pathways can be modulated in the elderly as a therapeutic strategy against NAFLD remains to be understood.

\section{Mitochondrial Dysfunction and Reactive Oxygen Species Production}

Mitochondrial respiration is often impaired in the livers of those over 50 years of age. In a previous study of 107 liver specimens, the majority $(87 \%)$ of respiratory chain defects occurred in those aged $>50$ years. ${ }^{23}$ Interestingly, mitochondrial defects were more prevalent in cirrhotic livers when compared with healthy livers $(78 \%$ vs $57 \%)$. The main affected respiratory chain protein complex in the mitochondrion is complex IV of the respiratory chain, which is assembled by proteins encoded by both nuclear and mitochondrial DNA. ${ }^{24}$ In aged mouse and rat models, mitochondria isolated from the liver also displayed a decrease in activity of complex I, II, IV, and V. Additionally, older rats have larger mitochondria with lower membrane potential, which are less efficient in producing ATP compared to those of younger rats. ${ }^{25-28}$ Mitochondria are the main endogenous sources of ROS, which are generated as a byproduct of cellular respiration. ${ }^{29}$ ROS can cause damage to various cellular macromolecules such as proteins, lipids, and nucleic acids. Because the production of ROS increases with age, the ROS-mediated accumulation of cellular damage is postulated to be one of the causes of cellular senescence and aging-related decline. ${ }^{30}$ In the liver, the same basic principle may occur: liver mitochondria from aged mice produce higher levels of ROS and have higher degrees of mitochondrial dysfunction when compared with younger animals. ${ }^{31}$ In patients with NASH, the liver-specific activities of the respiratory chain complexes are lower than those observed in healthy patients, and it has been postulated that the mitochondrial dysfunction and increased oxidative stress mediate the progression of NAFLD to $\mathrm{NASH},{ }^{32,33}$ potentially even more so in elderly patients.

\section{Prevalence and Natural History of NAFLD \\ Prevalence of NAFLD}

Metabolic diseases and NAFLD have an intertwined and complex relationship. On the one hand, metabolic diseases can develop as a consequence of NAFLD, and on the other hand, different metabolic diseases (such as central obesity, T2DM, dyslipidemia, and insulin resistance) are risk factors for developing NAFLD. Obesity is also related to NAFLD severity, but interestingly, NAFLD can also be found in nonobese patients, particularly in certain Asian countries. ${ }^{34}$ This 
particular manifestation of lean NAFLD is characterized by unique clinical characteristics, such as higher levels of liver transaminases, as well as insulin and a lower degree of insulin sensitivity. ${ }^{35}$ It is widely accepted that the early diagnosis of NAFLD is essential, to allow for proper management of those burdened by the disease. However, it can go undiagnosed for some time, increasing the chance of disease progression, and leading to serious complications, such as fibrosis, cirrhosis, or HCC.

The aforementioned risk factors can accumulate in elderly patients and thus, special attention must be made to monitor these patients for NAFLD and its advanced stages. In the elderly, NAFLD prevalence rates vary. Some studies report an "inverted U curve", ie, the prevalence reaching a peak during late adulthood and decreasing from there onward, resulting in older cohorts of patients with a lower prevalence of NAFLD than some younger cohorts. For example, in a study of Japanese patients, the prevalence among males plateaued between 40 and 49 years of age at around $45 \%$, and decreased to values close to $25 \%$ in those aged 70 or older. In females, however, the prevalence of NAFLD reached a maximum of $31 \%$ in those aged $60-69$ years old, and decreased to $20 \%$ in those $\geq 70$ years of age. ${ }^{36}$ In another study focusing on 3271 elderly individuals from the US, the pooled prevalence (males and females) was slightly lower in those aged 75 or over when compared to the younger cohort encompassing patients between 60 and 74 years of age (39.2\% vs $40.3 \%$, respectively). ${ }^{37}$ However, not every study observes an "inverted U" curve. A meta-analysis assessment of the global prevalence of NAFLD observed a consistent increase of the prevalence value across all age groups from 30-39 through to 70-79 years old. Nevertheless, the value reported for the cohort $>70$ years was obtained from a single study, which may introduce some bias. ${ }^{38}$ Whether the lower prevalence in older generations is due to a selection effect caused by patients with NAFLD dying earlier than those without NAFLD or due to lifestyle differences between older and younger generations must be addressed in future studies.

\section{Natural History of NAFLD in the Elderly}

NAFLD slowly progresses over years and only a subset of patients progress to NASH with liver fibrosis. ${ }^{39}$ A recent retrospective study specific to US patients demonstrated that the progression of simple NAFLD/NASH to more severe liver disease (namely cirrhosis, liver transplant, or HCC) was $9 \%$ after 1 year, reaching $39 \%$ at year 8 post- diagnosis. ${ }^{40}$ A previous study using paired biopsies had shown that in patients diagnosed with fatty liver, $44 \%$ were observed to progress to NASH in a follow-up biopsy, while $22 \%$ progressed to stage 3 fibrosis; the median interval between the initial and follow-up biopsy was 6.6 years. Importantly, the fibrosis stage is an important predictor of all-cause and liver-related mortality in patients with NAFLD. Particularly, when compared with a reference population of patients without fibrosis (stage 0), those with stage 1 fibrosis had elevated allcause and liver-related mortality rate ratios (MRRs) of 1.58 and 1.41 , with the latter increase not being statistically significant. Both MRRs increased with the fibrosis stage, reaching values of 6.4 and 42.3, respectively, for patients with stage 4 fibrosis. ${ }^{41}$ While it is accepted that the risk of mortality increases with higher stage of liver fibrosis, ${ }^{42-44}$ the absolute increase(s) and the cohorts at risk differ between studies. For example, two previous studies showed that only patients with stage 3 and/or 4 fibrosis had an increased risk of all-cause mortality, while those with less severe fibrosis were spared. ${ }^{43,44}$ Regarding liver-related mortality, increased mortality had also been reported only in patients with stage 2 (or higher) fibrosis, but the absolute values ranged from 5 - to 47 -fold increased risk in patients with stage 4 fibrosis. ${ }^{44}$ Importantly, the disparate results could be a result of biases introduced by the selection criteria of studies focusing on the natural history of NAFLD. Given that biopsy confirmation is usually required as an inclusion criterion, the underlying reason for the biopsy is important: if it is performed to clarify a specific clinical suspicion (such as disease progression), the study population may consist of patients with a more severe form of the disease, that are more likely to experience negative outcomes.

There are only a limited number of studies reporting the outcomes of NAFLD specifically in elderly cohorts. As in the general population, the course of the disease is not very well understood in this cohort of patients. One of the earlier studies highlighted the elevated prevalence of NAFLD in octogenarians. ${ }^{45}$ The prospective study, enrolling a total of 91 patients aged 80 years or older, revealed that almost half (42 out of 91) had NAFLD. When comparing both groups, the authors observed that the prevalence of metabolic syndrome (or any of its diagnostic criteria) was not modified by the presence of NAFLD. ${ }^{45}$ These findings suggested that NAFLD is both common and benign in the elderly. However, the possibility of a "survivorship bias" was also raised, suggesting that the 
subset of older adults with more severe complications arising from NAFLD may have died earlier, contributing to biases in the results. Two studies published afterward confirmed the "survivorship bias" hypothesis to be plausible. First, in an Asian cohort, those $<60$ years of age were less likely to have severe steatosis; at the same time, $\geq 60$ years of age was also associated with more severe (degree 3 or 4) fibrosis. ${ }^{46}$ Second, a larger cohort of patients from a tertiary care clinic in the UK showed older patients with NAFLD to have a higher burden of risk factors for the development of NAFLD, namely hypertension, diabetes, hyperlipidemia, or obesity. The fibrosis score was also significantly higher in older ( $\geq 60$ years of age) patients. The levels of liver-related serum markers were also suggestive of more severe liver disease in the older cohort of patients. Importantly, the authors did not observe a difference in terms of comorbidities between the different age groups, suggesting that the more severe disease in older patients is directly related to NAFLD. ${ }^{1}$ A more recent study directly compared the outcomes of NAFLD in younger and older elderly patients ( $60-74$ vs $\geq 75$ years old, respectively) and confirmed that NAFLD was associated with higher all-cause and cardiovascular-related mortality exclusively in those aged 60-74 years old. In the older group of patients, no statistically significant differences were observed between patients with and without NAFLD. As briefly mentioned before, patients with a more severe form of NAFLD may be more likely to die before the 7th or 8th decade of life; this underlines that the groups of patients $\geq 70$ years old who are included in epidemiological studies are "enriched" in individuals with more benign forms of NAFLD. Alternatively, as females are protected from NAFLD until postmenopausal ages, the natural course of the disease may have a "lag period" when compared to males. ${ }^{47}$ Indeed Golabi et al demonstrated that patients $\geq 75$ years old with NAFLD were predominantly female. ${ }^{37}$ Therefore, the authors postulated that the more benign nature of NAFLD may be explained by the earlier death of males who had been afflicted by the disease for longer than their female counterparts. To the best of our knowledge, no other studies exist which permit deeper analysis of the natural history of NAFLD in the elderly, particularly in those $>70$ years of age. Given that NAFLD decreases life expectancy by $\sim 4$ years, the disease must be detected as early as possible to reduce disease-related mortality. ${ }^{48}$ In the future, larger studies that carefully address potential sources of bias associated with the inclusion of older adults are necessary. Further, given that women may only be afflicted by NAFLD later in life, it will be essential to perform analyses between sex-matched cohorts. The resulting data should help optimize current treatment and diagnosis guidelines.

\section{NAFLD and Related ExtraHepatic Diseases}

NAFLD emerges in the context of comorbidities, that intertwine and influence each other adding to the complexity of the disease. ${ }^{49}$ Besides the potentially life-threatening hepatic consequences of NAFLD, such as end-stage cirrhosis or HCC, NAFLD impacts the renal, cardiovascular, endocrine, and skeletomuscular systems. ${ }^{50}$ Given that most of these systems also experience age-related changes, it is important to consider the interplay between "aged" biological systems and NAFLD. Therefore, it is beneficial for both physicians and elderly patients to consider the multiple organ involvement of NAFLD.

\section{Hepatocellular Carcinoma}

The association between NAFLD and HCC is widely accepted. The incidence rate of HCC among patients with NAFLD and cirrhosis is estimated at $6.7 \%$ and $15 \%$ over 5 and 10 years, respectively. However, in the absence of cirrhosis, the incidence rate is as low as 23 per 100,000 person-year, despite values as high as $2.7 \%$ over 10 years having been reported as well. ${ }^{51}$ Interestingly, multivariate regression analysis pointed to older age as an independent variable associated with developing HCC in patients with NASH-associated cirrhosis. ${ }^{52}$ Another study has also established advanced age as a risk factor for HCC in patients with NASH and liver cirrhosis. ${ }^{53}$

In elderly patients, HCC tends to be associated with less severe fibrosis than when detected in younger patients. It is also more likely to be encapsulated and, consequently, to have a more favorable prognosis. ${ }^{54,55}$ In older patients, a series of age-related concerns can be raised that may undermine the benefits of surveillance for HCC, such as exclusion from transplantation programs and extrahepatic comorbidities. ${ }^{56}$ However, in a study focusing on elderly Italian patients with cirrhotic NAFLD, it was shown that active HCC surveillance reduced mortality. ${ }^{56}$ Therefore, physicians should not disregard the benefits of surveillance in this cohort of patients. Due to the lower incidence rates of HCC in the absence of cirrhosis, HCC surveillance is currently only recommended in patients with NAFLD, who exhibit advanced fibrosis. ${ }^{57}$ As mentioned above, 
advanced age is a risk factor for the development of HCC. In Taiwan, the incidence rate of HCC has steadily increased from 2003 to 2011 in patients 65 years of age or older and this increase was presumed to be associated with the increased prevalence of NAFLD. ${ }^{58}$ Similar trends were observed in the US between 2000 and 2009. ${ }^{59}$ Therefore, active HCC surveillance may be warranted in the elderly, even in the absence of fibrosis. Importantly, other factors such as viral hepatitis and alcoholic cirrhosis, rather than NAFLD, can also lead to the development of HCC. $^{60}$ Accordingly, more studies will be necessary to establish the degree NAFLD contributes to the development of HCC in the elderly and to what extent the diagnosis of NAFLD without associated cirrhosis warrants active HCC surveillance in this particular population.

\section{Type 2 Diabetes Mellitus}

Perhaps unsurprisingly, two meta-analyses showed that the prevalence of NAFLD in patients with T2DM is around 50 $-60 \% .{ }^{61}$ Likewise, NAFLD increases the risk of T2DM by over 2-fold and was higher in patients with more severe NAFLD. ${ }^{62}$ This two-way relationship between both diseases is also illustrated by a higher prevalence of diabetic retinopathy and nephropathy in T2DM in patients with concomitant NAFLD.$^{63}$ Importantly, the potential interaction between both diseases is more relevant in aged adults, as the prevalence of NAFLD among adults with T2DM increases with age. In a meta-analysis of 80 studies, the global prevalence of NAFLD among T2DM patients was, similar to what was described above, $\sim 55 \%$. However, this value raised to $62.8 \%$ in those $\geq 60$ years of age. ${ }^{61}$ In a retrospective study of elderly Chinese patients, those with NAFLD at baseline experienced a higher incidence of both diabetes and prediabetes, with adjusted relative risk ratios of 1.67 and 1.34, respectively. ${ }^{64}$ Therefore, these patients are at higher risk of morbidity and mortality and should be managed accordingly.

\section{Cardiovascular and Coronary Heart Disease}

CVD is the most common cause of death in patients with NAFLD, and patients with NASH are roughly 2-times more likely to die from CVD compared to the general population. ${ }^{65}$ As with T2DM, the contribution of NAFLD to CVD risk is hard to determine, given that both diseases share common risk factors. ${ }^{66}$ For example, in a prospective observational study of Japanese individuals, NAFLD arose as a predictor of CVD, with an odds ratio of 4.12. NAFLD, but not the metabolic syndrome, was also associated with cardiovascular events. ${ }^{67}$ In a larger study, NAFLD per se was not associated with CVD. Instead, NAFLD with advanced fibrosis was a predictor of mortality, primarily from cardiovascular events. ${ }^{68}$ In this context, studies using magnetic resonance elastography to characterize the liver disease stage and pair those findings with coronary artery calcium scoring as a surrogate of CVD have observed an association starting at intermediated stages of fibrosis. ${ }^{69}$ Not all studies observed an association between both parameters, though. Stepanova et al demonstrated that NAFLD increased the risk of CVD, but not CVD-related mortality. ${ }^{70}$ Similarly, Lazo and colleagues also failed to observe an increased risk of CVD mortality in patients with NAFLD. ${ }^{71}$ Further, it contributes to the release of inflammatory and fibrogenic mediators, resulting in cardiac complications. NAFLD has been associated with endothelial dysfunction, increased coronary arterial calcifications, and increased carotid thickness, which are all markers of CVD. Importantly, the CVDs introduced or exacerbated by NAFLD are all common in the elderly and this topic has been extensively discussed elsewhere. ${ }^{66,72}$

\section{Microbiota, Age and NAFLD}

All surfaces of the human body are covered by a complex ecosystem of microorganisms as bacteria, archaea, viruses, and fungi. The totality of these microorganisms in one habitat is defined as the microbiome. ${ }^{73}$ Due to the strategic position at the interface between host and environment, the microbiome acts as a metabolically active organ and functions as the turning point for the integration of environmental signals. ${ }^{74}$ The individual microbiota signature in combination with host genetics contribute to a person's distinct characteristics and its specific phenotype. During adulthood, the microbiota at the species level is relatively stable, but decreases with increasing age. ${ }^{75,76}$ Larger interindividual variability is often observed in the elderly. These changes might be related to differences in lifestyle and dietary habits. ${ }^{77}$ Age-related changes in taste and smell and swallowing difficulties may impact the diet. ${ }^{78}$ A reduced intake of fiber and proteins can lead to changes in the composition of the microbiota. ${ }^{79}$ Moreover, lack of vitamin $\mathrm{D}$, calcium and protein can affect both the composition and activity of the microbiota. ${ }^{80}$

With increasing age, the gut-associated lymphoid tissue decreases, and the innate immune defenses grow less efficient. These changes are often responsible a chronic, low grade overall inflammatory status. ${ }^{81}$ Moreover, in 
elderly the cellular immune function decreases. This immunosenescence may lead to increased importance of antibody-associated responses in the elderly. The resulting surplus of antibodies is reflected in increased autoimmunity. ${ }^{82}$

While it is well known that obesity is the most important risk factor for the development of NAFLD, recent studies highlighted the gut-liver axis and especially the intestinal microbiota play a key role in NAFLD development progression. ${ }^{83}$ Therefore, an unfavorable microbiota in elderly will not only influence the liver metabolism. ${ }^{84}$ Moreover, it modulates the inflammatory environment in the liver through microbiota derived molecules and metabolites, reaching the liver via the portal vein. Studies including NASH patients showed reduced diversity of microbiota species, and a changed ratio of bacteroidetes and firmicutes bacterial groups. ${ }^{85,86}$ Interestingly, a decrease in Firmicutes has also been reported in the Italian population over 60 years. Similarly, an age-related increase in Bacteroidetes was found in elderly subjects from Northern Europe. ${ }^{76,87}$ Therefore, microbiota changes may play a role in future approaches to target NAFLD in elderly.

\section{Sarcopenia and NAFLD in Elderly}

Sarcopenia is defined by reduced muscle mass and replacement of muscle with adipose or fibrous tissue. ${ }^{88}$ NAFLD and Sarcopenia share several pathophysiological mechanisms: Both are associated with metabolic changes as insulin resistance. ${ }^{89}$ Next to the well-known effects of insulin sensitivity in NAFLD, it is in Sarcopenia a key mediator of muscle loss. ${ }^{90}$ Another link of NAFLD and Sarcopenia is Vitamin D. A potential pathophysiological substratum involving joint molecular pathways is highly probable, but not yet shown. ${ }^{91}$

Lastly, inflammation also links sarcopenia to NAFLD. The association between NAFLD and inflammation and inflammation and sarcopenia, is well known. ${ }^{92}$ On one hand, important indicators of inflammation, such as TNF$\alpha$, IL-6 and CRP, are associated with both, sarcopenia and NAFLD. ${ }^{92}$ On the other hand, inflammation is apparently related to insulin sensitivity. ${ }^{92}$

\section{Autonomic System Dysfunction and NAFLD in Elderly}

With increased age changes in the autonomic nervous system can occur. Those changes can be measured by using the heart rate variability (HRV), a non-invasively measured parameter that measures the cardiac autonomic function. ${ }^{93}$ Symptoms of cardiac autonomic dysregulation are vasovagal syncope, fatigue, or orthostatic hypotension. Changes in the autonomic nervous system are related to the metabolic syndrome, which may be a link to NAFLD.${ }^{94}$ It is gradually recognized, however, NAFLD patients report a wide-ranging spectrum of non-specific symptoms, such as fatigue and daytime sleepiness, which may be the presenting complaint and may dramatically impact quality of life. ${ }^{95}$ Moreover, a high prevalence of autonomic dysfunction in patients with chronic liver disease is described. ${ }^{96}$ Fatigue is an often-reported comorbidity in NAFLD. While the severity of fatigue does not mirror the severity of histological changes, it is associated with daytime sleepiness and autonomic dysfunction. ${ }^{95}$ Autonomic dysfunction can lead to severe clinical consequences such as cognitive dysfunction, falls and fallrelated injuries. ${ }^{95}$

\section{Cognitive Decline and NAFLD in Elderly}

Another feature of pathological aging is cognitive decline. It is well known that end stage liver disease is associated with encephalopathy. Still, it is speculated that earlier stages of liver diseases might be associated with some form of cognitive decline: Hepatitis $\mathrm{C}$ and primary biliary cirrhosis have been associated with neuropsychological impairment even in earlier disease stages. ${ }^{97,98}$ In addition, NAFLD has been proposed to be an additional risk factor for dementia. ${ }^{99}$ Then mechanisms are still unknown, but it is speculated that the association of NAFLD and carotid atherosclerosis may lead to cognitive decline. ${ }^{100}$ Separately from the apparent effects of cerebrovascular disease on cognitive decline, hormonal alterations that are associated with NAFLD and especially insulin resistance may further spur the cognitive decline. ${ }^{101}$ Moreover, low levels of vitamin B12 are associated with both NAFLD and negative cognitive consequences. ${ }^{102}$ Although there are no studies that compare NAFLD, dementia and lack of vitamin B12, the presence of a pathway involving dementia with NAFLD seems plausible.

\section{Adipose Tissue and NAFLD in Elderly}

With increasing age not only the amount of body fat changes, the fat distribution shifts from mostly subcutaneous to visceral fat. ${ }^{103}$ Increased visceral fat is associated with increased cardiovascular risk, insulin resistance and the metabolic syndrome. In addition to the negative effects of 
visceral fat accumulation, the age-related reduced capacity of subcutaneous fat cells to store lipids can lead to negative metabolic effects via lipotoxicity. ${ }^{104}$ In elderly the number of preadipocytes, which are fat cell progenitors, decline. ${ }^{105}$ This may account for an imbalance between lipolysis and lipogenesis, which in turn leads to an increased exposure to free fatty acids and enhances lipotoxicity. ${ }^{106,107}$ These changes translate into oxidative stress and increased production of proinflammatory cytokines. ${ }^{108}$ Fat cell senescence is accompanied by alterations in fat cell biochemistry, proliferative capacity, and immune response. ${ }^{104}$ In NAFLD in elderly, these changes together with age-related hormonal changes in GH as well as IGF-1 and the related activation of proinflammatory cytokines may play a causal role. ${ }^{106}$ These crucial implications of adipose tissue and fat distribution in elderly, is highlighted by a recent study that found that reducing abdominal fat, and not only BMI, in improves survival in the elderly. ${ }^{109}$

\section{Diagnosis and Screening of NAFLD}

As it stands today, diagnosing NAFLD requires the exclusion of secondary causes of hepatic fat or harmful alcohol, usually by imaging or (ideally) histological staging. ${ }^{110}$ The systematic screening for NAFLD in the general population is not recommended. However, people at risk of advanced fibrosis, in particular patients living with T2DM should be explored if elevated liver function tests or hepatic steatosis are present according to the ADA. ${ }^{111}$ Some concerns include the costs of testing, the risk of liver biopsy, and the lack of effective treatments. However, it is reasonable to suggest that systematic screening or vigilance for NAFLD should be promoted in high-risk individuals.

\section{Noninvasive Methods}

Liver enzymes have a low positive predictive value and are not an accurate predictor of NAFLD. Up to $80 \%$ of patients with NAFLD exhibit normal levels of liver enzymes, while patients with advanced liver disease may have lower levels of alanine aminotransferase (ALT) ${ }^{112,113}$ In patients with NAFLD and normal ALT levels, the histological profile of the liver is similar to those with abnormal levels of other liver enzymes. ${ }^{114}$ This may be related to the fact that NAFLD remains asymptomatic until it progresses to advanced liver disease when treatments or prevention are rendered less or non-effective. While these tools are not specific for elderly patients, we will briefly describe the main currently available scoring systems in use.

\section{Scoring Systems}

The NAFLD Liver Fat Score (NLFS) aims to quantify the degree of fat in the liver by attributing scores to different parameters: metabolic syndrome, fasting insulin level, T2DM, aspartate aminotransferase (AST), and AST/ALT ratio. By using a cut-off point of -0.640 , NFLS predicts increased liver fat content with a sensitivity of $86 \%$ and specificity of $71 \%{ }^{115}$ The Fatty Liver Index (FLI) is a score that, instead, uses parameters such as BMI, waist circumference, gamma-glutamyltransferase, and triglycerides. In a Chinese cohort, it had a sensitivity of $80 \%$ and a specificity of $72 \%$ for the diagnosis of NAFLD. ${ }^{116}$ It appears to be slightly superior to the Hepatic Steatosis Index (HIS). The second is more similar to NLFS in the sense that it includes which includes the AST/ALT ratio, BMI, T2DM, and sex. It has a reported sensitivity of $86 \%$ and a specificity of $66 \% .^{117}$

Currently, no elderly-specific recommendations exist regarding the diagnosis of NAFLD or NASH. ${ }^{110,118}$ However, recent evidence suggests that fibrosis scoring systems require different cut-offs in elderly patients. Initial studies assessing the utility of these scores focused primarily on middle-aged patients, did not include elderly patients or failed to determine the diagnostic cut-off values in this cohort of patients. ${ }^{119-122}$ Because both NFS and FIB-4 take age into account, there is an inflation of the score in the elderly due to the inclusion of this parameter by itself. As a result, more patients are considered to be intermediate-risk in virtue of their age, which increases the false positive rate. ${ }^{121}$ McPherson et al show that by using standard cut-off values, the specificity of FIB4 and NFS for advanced fibrosis declines with age, plummeting to $35 \%$ and $20 \%$, respectively in those aged $\geq 65$ years. By adjusting the cut-off values, specificity was improved to $70 \%$ for both scoring systems, while sensitivity remained above $75 \%$. In a recent study, these cut-offs were tested and the low specificity confirmed in elderly Asian patients. ${ }^{122}$ By using the new age-adjusted cut-offs proposed by McPherson, specificity was increased albeit at the cost of lower sensitivity. Therefore, more studies are necessary to understand how to employ fibrosis scoring systems while taking the patient's age into account.

\section{Imaging Tools}

Fat content and liver stiffness can be indirectly measured noninvasively through four mainstay techniques: magnetic resonance imaging (MRI), computed tomography, ultrasonography, and transient elastography. ${ }^{123}$ The first 
three techniques can be used to measure liver steatosis, but ultrasound only allows for qualitative evaluation. The sensitivity of those techniques varies between 75 and $80 \%$, while the specificities range from 85 to $100 \%{ }^{124}$ On the other hand, elastography imaging can be used both to diagnose steatosis, and degree of fibrosis with the sensitivity of different modalities of this technique ranging from 80 to $90 \% .^{125}$ As an advantage over the above-mentioned techniques, elastography can be used to diagnose advanced fibrosis with a sensitivity $>90 \%$ and specificity ranging from 75 to $92 \%{ }^{126}$

\section{Liver Biopsy}

Liver biopsy is the gold standard for the diagnosis of NAFLD and consequent evaluation of histological abnormalities in patients with the disease. It is generally limited by its cost and a high potential for sampling error, along with a high risk of complications; further, it is poorly accepted by patients. ${ }^{123}$ Current guidelines recommend that liver biopsy is reserved for patients at risk of steatohepatitis or advanced fibrosis, as predicted by noninvasive methods such as NFS and/or FIB4, in the context of clinical trials or indeterminate cases that require staging. It should also be considered when the presence or severity of the chronic liver disease cannot be ascertained without performing a biopsy. Based on current evidence, liver biopsies should not be avoided in the elderly purely for the sake of their advanced age. Almost 1 out of 15 liver biopsies in England and Wales are carried out in patients aged 80 or above, with a similar mortality rate as those performed in younger patients. ${ }^{127}$ However, more recent retrospective studies focusing on different populations should be taken into account before a specific recommendation is made. The healthcare team should carefully weigh the risks and benefits before deciding to perform a liver biopsy in an elderly patient.

\section{Diagnosing NAFLD Renamed as MAFLD in Elderly}

Many objections have been voiced about the nomenclature and definition of NAFLD regarding the notable role that alcohol plays in the definition and the negative consequences of the nomenclature, including trivialization. In medicine, communication matters, and the expressions used to name a disease can also profoundly impact patients. Lately, a consensus of international experts recommended that the disease acronym be changed from
NAFLD to metabolic dysfunction associated fatty liver disease or 'MAFLD. ${ }^{128}$ This change goes far beyond a semantic change and may be the first step that catalyzes the method to conceptualize better the condition for health promotion, patient orientation, case identification, ongoing clinical trials, and for the health services delivery. ${ }^{129}$

The suggested redefinition of the condition, especially in elderly, can increase patient awareness and understanding and garner funding more effectively for research efforts to mitigate the impacts of the MAFLD.

\section{Treatment of NAFLD}

The management of NAFLD is multifaceted: the underlying liver disease must be treated in parallel with the associated extrahepatic and systemic abnormalities, such as obesity, hyperlipidemia, and insulin resistance. ${ }^{110,118}$ From this perspective, two avenues of treatment are considered: lifestyle modifications and pharmacological treatment. Based on the notion that treatment is only appropriate if it improves outcomes, current guidelines recommend that owing to the overall good hepatic prognosis of patients without steatohepatitis, pharmacological treatments aimed primarily at improving liver disease should generally be limited to those with a high risk of progression to cirrhosis or decompensation of the underlying liver disease. ${ }^{110,112}$ The aspects in the elderly patient with NAFLD are summarized in Figure 2.

\section{Lifestyle Modifications}

Dietary modifications, more exercise, and weight loss are the main cornerstones of dietary modifications suggested to treat patients with NAFLD. Ultimately, the goal is to achieve weight loss, which has been shown to improve the histological features associated with NASH and the level of steatosis along with the liver chemistry. ${ }^{130}$ In a prospective trial lasting 12 months, using paired liver biopsies from 261 patients, the amount of weight lost correlated positively with the improvement of histopathological features. ${ }^{131}$ More than half $(58 \%)$ of the patients who achieved over 5\% weight loss experienced the resolution of $\mathrm{NASH}$, coupled with a reduction of the NAFLD activity score (NAS) compared to subjects who lost $<5 \%$ of their body weight. All patients who lost over $10 \%$ body weight showed a reduction of the NAS-Score, and $90 \%$ experienced a resolution of NASH. Almost half (45\%) had a regression of fibrosis, which is striking given the relatively short duration of the study (12 months). Similarly to what was described above, only $50 \%$ of the patients 


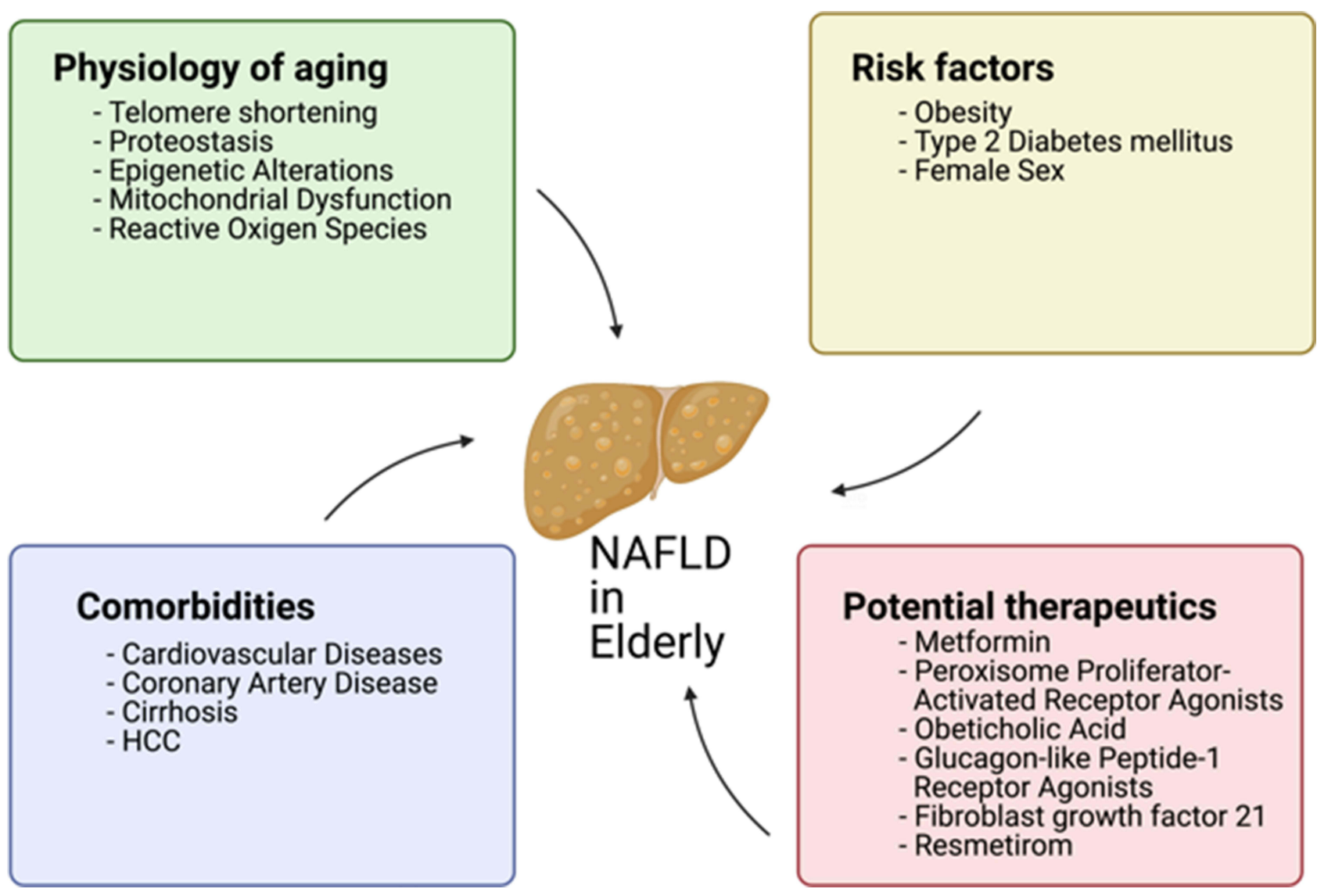

Figure 2 Clinical Features of NAFLD in elderly. Created with BioRender.com

achieved a weight loss equal or superior to $7 \%$ of their body weight. Therefore, despite the excellent outcomes, compliance with a calorie restriction dietary regimen is still a major obstacle to tackle from a physician's perspective. ${ }^{132}$ Interestingly, compliance with weight loss lifestyle modifications are generally better accepted by older patients. ${ }^{133,134}$ Patients with NAFLD are generally more sedentary, and a sedentary lifestyle is associated with NAFLD development and progression. ${ }^{135}$ Generally, the combination of exercise and caloric restriction is advisable, ${ }^{118}$ as there is some evidence that exercise seems to improve the levels of liver enzymes and hepatic steatosis. ${ }^{136}$ Importantly, any lifestyle modification(s) should be introduced with a focus on long-term adherence. Therefore, the needs of each individual and the characteristics of the patient shape the ideal approach. In elderly, it is particularly important to take the safety and the individual state of the patient for lifestyle changes into account. In some patients these may be incompatible with certain levels of activity or exercise that would otherwise be tolerable by a younger person. It is especially important to ensure that the dietary instructions introduced are not excessively aggressive, given the special need for adequate nutrition in the elderly. ${ }^{137,138}$

\section{Pharmacological Treatment}

No drug is currently approved for the treatment of NASH. Accordingly, no specific recommendations are made in practical guidelines. ${ }^{118}$ Therefore, there are also no specific recommendations for the use of any drugs in elderly patients. In this section, we will mainly discuss ongoing clinical trials and the inclusion (or not) of an adequate group of elderly patients that can potentially generate data that may guide specific clinical indications in the future.

\section{Metformin}

Metformin is a well-tolerated insulin sensitizer, frequently used in patients with T2DM to reduce peripheral insulin resistance, downregulate gluconeogenesis, and reducing blood glucose levels by suppressing hepatic glucose production. ${ }^{139}$ An array of clinical trials have tested the efficacy of metformin in NAFLD, with varying degrees of success. A meta-analysis concluded that metformin did not 
improve NASH-related outcomes, despite improving liver function, BMI, and insulin resistance to some extent. ${ }^{140}$ This lack of improvement in liver histology led to the current consensus that metformin is not advisable to specifically treat NAFLD in patients of all ages. ${ }^{118}$

\section{Peroxisome Proliferator-Activated Receptor Agonists}

Thiazolidinediones, such as rosiglitazone and pioglitazone are another class of drugs that can be used in patients with T2DM. ${ }^{141}$ Because thiazolidinediones can reverse both adipose tissue dysfunction and insulin resistance in patients with obesity and $\mathrm{T} 2 \mathrm{DM},{ }^{142}$ some clinical trials have also assessed their potential benefits in the treatment of NAFLD. ${ }^{143}$ In a recent prospective study enrolling patients with biopsy-proven NASH with or without T2DM, pioglitazone reduced liver fibrosis and increased insulin sensitivity in both, patients with and without T2DM. However, the benefits were more pronounced in diabetic patients. ${ }^{144}$ Importantly, this clinical trial was limited to adults up to 70 years of age, and previous studies did not explicitly study the effect of thiazolidinediones on elderly cohorts. ${ }^{145,146}$ Given that thiazolidinediones are associated with a higher risk of heart failure and bone fractures, ${ }^{147,148}$ care must be exerted when considering these drugs in the elderly. ${ }^{149,150}$ Therefore, it is important to be particularly aware of the potential added risk of administering thiazolidinediones to the elderly.

Lanifibranor is a non-thiazolidinedione PPAR agonist currently being tested in the NATIVE study. Results are not yet published but the trial did not exclude older adults and did not have an upper age cut-off, thus, the data may potentially be used as a steppingstone for further studies addressing the efficacy of lanifibranor in elderly patients.

\section{Farnesoid X Receptor Agonist - Obeticholic Acid}

Obeticholic acid (OCA) is a synthetic derivative of chenodeoxycholic acid, with a 100-fold higher affinity for the Farnesoid X receptor (FXR) than the endogenous molecule. OCA has anticholestatic and hepatoprotective effects in an FXR-dependent fashion. ${ }^{151}$ OCA can increase sensitivity to insulin, regulate lipid and glucose metabolism, and exert an antifibrotic effect in the liver. ${ }^{152}$

Initially, the FLINT trial observed that OCA improved both the biochemical and histological profiles as well as the NAFLD activity score of patients with non-cirrhotic NASH after 72 weeks of OCA treatment compared to placebo. ${ }^{153}$ Over one-third $(35 \%)$ of the patients in the
OCA group experienced a regression of fibrosis when compared to only $19 \%$ in the placebo arm. A larger trial (REGENERATE) followed, based on the promising results of the FLINT trial. REGENERATE enrolled 1968 patients with fibrotic NASH, who had been receiving a lower $(10 \mathrm{mg} /$ day $)$ or higher $(25 \mathrm{mg} /$ day $)$ dose of OCA or placebo. ${ }^{154}$ After 18 months, the intention-to-treat (ITT) and the per-protocol (PP) populations registered an improvement of fibrosis with no worsening of NASH (ITT: $12 \%, 18 \%$, and $23 \%$ in the placebo, lower dose, and higher dose of OCA respectively; PP: $13 \%, 21 \%$, and $28 \%$ in the placebo, lower dose, and higher dose of OCA respectively). Pruritus and increased LDL cholesterol were the most common adverse effects. Importantly, both trials enrolled adults without an upper limit of age, but neither investigated the outcomes according to age. In the future, the data from these trials could potentially be analyzed across different age cohorts, with a particular focus on elderly cohorts. This information would help physicians to understand the risk/benefit ratio of OCA in older patients.

\section{Glucagon-Like Peptide-I Receptor Agonists}

Glucagon-like peptide-1 (GLP-1) analogs are an alternative therapeutic avenue undergoing active research in the context of NAFLD treatment. They exert a myriad of effects on organismal metabolism and can affect body weight, glucose and lipid homeostasis, as well as inflammation. ${ }^{155,156}$ Liraglutide and semaglutide are two GLP-1 mimetics that have been tested in patients with NASH. In the Phase 2 clinical trial LEAN, which enrolled obese patients with NASH, 9 out of 23 patients receiving liraglutide had resolution of NASH, compared to 2 of 22 patients in the placebo group. ${ }^{157}$ Another phase 2 clinical trial, albeit with semaglutide, focusing on 320 patients with NASH and stage F1, F2, or F3 fibrosis, reported similar results. NASH resolution without worsening of fibrosis was achieved in $40 \%, 36 \%$, and $59 \%$ of the patients receiving $0.1,0.2$, or $0.4 \mathrm{mg}$ /day subcutaneous semaglutide, respectively, when compared to just $17 \%$ in the placebo group. ${ }^{158}$ Nevertheless, these results may not apply to older adults, as the age cut-offs in the liraglutide and semaglutide trials were 70 and 75 years, respectively.

\section{Fibroblast Growth Factor 21}

Fibroblast growth factor 21 (FGF21) belongs to the family of fibroblast growth factors, with a myriad of systemic metabolic effects reviewed elsewhere. ${ }^{159}$ As with many 
other candidate drugs, it can reverse obesity and insulin resistance. ${ }^{159}$ The native form of FGF21 has a short halflife and tendency to aggregate. Accordingly, there has been an interest in generating more pharmacologically appealing derivatives to be used for the treatment of NAFLD. ${ }^{160}$ For example, pegbelfermin (BMS-986036) is a PEGylated form of FGF21 which has been employed in a phase 2 a trial. Daily and weekly administration of pegbelfermin led to a decrease in the absolute hepatic fat content when compared to placebo $(-6.8 \%$ and $-5.2 \%$, respectively. vs $-1.3 \%$ ). In the coming years, new clinical trials evaluating pegbelfermin should provide further results that will help elucidate the efficacy (or lack thereof) of this drug in the management of NASH-related complications. Unfortunately, two upcoming trials (FALCON 1 and 2) have established an upper age limit of 75 years old for patient inclusion. Therefore, limited conclusions specific to older adults can be drawn. ${ }^{161,162}$

\section{Resmetirom (MGL-3196)}

Resmetirom, also known as MGL-3196, is a thyroid hormone receptor $\beta$ agonist. ${ }^{163}$ The results of its phase 2 trial were recently published, enrolling adults with biopsyconfirmed NASH and stage 1 to 3 fibrosis, along with a hepatic fat fraction $\geq 10 \%$. Resmetirom-treated patients showed a relative reduction of hepatic fat compared with placebo at week $12(-32.9 \%$ vs $-10.4 \%)$ and week 36 $(-37.3 \%$ vs -8.5$)$. On secondary analyses, NASH resolution was achieved in a higher proportion of treated patients and was correlated with higher doses and increased exposure to the drug. A Phase 3 trial enrolling 2000 patients is currently ongoing, in which older patients should be well represented (no upper age limit). ${ }^{164}$ The results will be invaluable to understand if resmetirom will be safe and effective in the elderly NASH population.

\section{Conclusion}

Aging is a natural process that requires adaptations in medical care and consideration for treatment. As the prevalence of NAFLD increases with age, and NAFLDrelated complications may represent an important source of morbidity and healthcare utilization, this disease should be addressed in the elderly population. The importance of comorbidities and polypharmacy is largely unaddressed in current clinical guidelines. Further, there is still controversy regarding which population should be actively screened for NAFLD and/or NASH. However, a lot of the controversy stems from the lack of adequate treatments, which limit the value of a diagnosis at current. It can be expected that when effective treatments become available, physicians should be more inclined to secure the diagnosis and initiate pharmacological treatment. Unfortunately, the elderly population is often not adequately represented in clinical trials and thus this will be an extra hurdle for physicians and patients deciding on whether a treatment's benefits outweigh the potential risks. Here, further epidemiological and mechanistic studies, along with an effort not to exclude older adults from clinical trials, will be necessary to understand the better approach to diagnose and treat NAFLD in older adults.

\section{Disclosure}

JMS reports Consultancy: Boehringer Ingelheim, BMS, Genfit, Gilead Sciences, Intercept Pharmaceuticals, Madrigal, Novartis, Novo Nordisk, Nordic Bioscience, Pfizer, Roche, Sanofi, Siemens Healthcare GmbH. Research Funding: Gilead Sciences, Boehringer Ingelheim. Speakers Bureau: Falk Foundation MSD Sharp \& Dohme GmbH. JMS is partly funded by the European Union Innovative Medicines Initiative 2 (IMI2) Joint Undertaking under grant agreement 777377: LITMUS (Liver Investigation: Testing Biomarker Utility in Steatohepatitis) and the "LiverScreen" project (grant agreement No 847989). SAA reports no conflicts of interest in this work.

\section{References}

1. Frith J, Day CP, Henderson E, Burt AD, Newton JL. Non-alcoholic fatty liver disease in older people. Gerontology. 2009;55(6):607-613. doi: $10.1159 / 000235677$

2. Munday D, Leaman J, O’Moore É, Plugge E. The prevalence of non-communicable disease in older people in prison: a systematic review and meta-analysis. Age Ageing. 2019;48(2):204-212. doi:10.1093/ageing/afy186

3. López-Otín C, Blasco MA, Partridge L, Serrano M, Kroemer G. The Hallmarks of aging. Cell. 2013;153(6):1194-1217.

4. Aikata H, Takaishi H, Kawakami Y, et al. Telomere reduction in human liver tissues with age and chronic inflammation. Exp Cell Res. 2000;256(2):578-582. doi:10.1006/excr.2000.4862

5. Singh R, Kaushik S, Wang Y, et al. Autophagy regulates lipid metabolism. Nature. 2009;458(7242):1131-1135. doi:10.1038/ nature 07976

6. Zhang C, Cuervo AM. Restoration of chaperone-mediated autophagy in aging liver improves cellular maintenance and hepatic function. Nat Med. 2008;14(9):959-965. doi:10.1038/nm.1851

7. Schneider JL, Villarroya J, Diaz-Carretero A, et al. Loss of hepatic chaperone-mediated autophagy accelerates proteostasis failure in aging. Aging Cell. 2015;14(2):249-264. doi:10.1111/acel.12310

8. Passos JF, Saretzki G, von Zglinicki T. DNA damage in telomeres and mitochondria during cellular senescence: is there a connection? Nucleic Acids Res. 2007;35(22):7505-7513. doi:10.1093/nar/gkm893 
9. Victorelli S, Passos JF. Telomeres and Cell senescence - size matters not. EBioMedicine. 2017;21:14-20. doi:10.1016/j.ebiom.2017.03.027

10. Campisi J, d'Adda Di Fagagna F. Cellular senescence: when bad things happen to good cells. Nat Rev Mol Cell Biol. 2007;8 (9):729-740. doi:10.1038/nrm2233

11. Rudolph KL, Chang S, Millard M, Schreiber-Agus N, DePinho RA. Inhibition of experimental liver cirrhosis in mice by telomerase gene delivery. Science. 2000;287 (5456):1253-1258. doi:10.1126/science.287.5456.1253

12. Aravinthan A, Mells G, Allison M, et al. Gene polymorphisms of cellular senescence marker p21 and disease progression in non-alcohol-related fatty liver disease. Cell Cycle. 2014;13 (9):1489-1494. doi:10.4161/cc.28471

13. Aravinthan A, Scarpini C, Tachtatzis P, et al. Hepatocyte senescence predicts progression in non-alcohol-related fatty liver disease. J Hepatol. 2013;58(3):549-556. doi:10.1016/j.jhep.2012.10.031

14. Nakajima T, Nakashima T, Okada Y, et al. Nuclear size measurement is a simple method for the assessment of hepatocellular aging in non-alcoholic fatty liver disease: comparison with telomere-specific quantitative FISH and p21 immunohistochemistry. Pathol Int. 2010;60(3):175-183. doi:10.1111/j.1440-1827.2009.02504.x

15. Verma S, Tachtatzis P, Penrhyn-Lowe S, et al. Sustained telomere length in hepatocytes and cholangiocytes with increasing age in normal liver. Hepatology. 2012;56(4):1510-1520. doi:10.1002/ hep. 25787

16. Wang M-J, Chen F, Li J-X, et al. Reversal of hepatocyte senescence after continuous in vivo cell proliferation. Hepatology. 2014;60(1):349-361. doi:10.1002/hep.27094

17. Ogrodnik M, Miwa S, Tchkonia T, et al. Cellular senescence drives age-dependent hepatic steatosis. Nat Commun. 2017;8(1):15691.

18. Cavalli G, Heard E. Advances in epigenetics link genetics to the environment and disease. Nature. 2019;571(7766):489-499. doi:10.1038/s41586-019-1411-0

19. Pogribny IP, Tryndyak VP, Bagnyukova TV, et al. Hepatic epigenetic phenotype predetermines individual susceptibility to hepatic steatosis in mice fed a lipogenic methyl-deficient diet. J Hepatol. 2009;51(1):176-186. doi:10.1016/j.jhep.2009.03.021

20. Shock LS, Thakkar PV, Peterson EJ, Moran RG, Taylor SM. DNA methyltransferase 1, cytosine methylation, and cytosine hydroxymethylation in mammalian mitochondria. Proce Nat Acad Sci. 2011;108(9):3630-3635. doi:10.1073/pnas.1012311108

21. Pirola CJ, Gianotti TF, Burgueño AL, et al. Epigenetic modification of liver mitochondrial DNA is associated with histological severity of nonalcoholic fatty liver disease. Gut. 2013;62 (9):1356-1363. doi:10.1136/gutjnl-2012-302962

22. Bacalini MG, Franceschi C, Gentilini D, et al. Molecular Aging of human liver: an epigenetic/transcriptomic signature. J Gerontolo. 2019;74(1):1-8.

23. Muller-Hocker J, Aust D, Rohrbach H, et al. Defects of the respiratory chain in the normal human liver and in cirrhosis during aging. Hepatology. 1997;26(3):709-719. doi:10.1002/ hep. 510260324

24. Lazarou M, Smith SM, Thorburn DR, Ryan MT, McKenzie M. Assembly of nuclear DNA-encoded subunits into mitochondrial complex IV, and their preferential integration into supercomplex forms in patient mitochondria. FEBS J. 2009;276(22):6701-6713. doi:10.1111/j.1742-4658.2009.07384.x

25. Bellanti F, Romano AD, Giudetti AM, et al. Many faces of mitochondrial uncoupling during age: damage or defense? J Gerontolo. 2013;68(8):892-902. doi:10.1093/gerona/gls332

26. Modi HR, Katyare SS, Patel MA. Ageing-induced alterations in lipid/phospholipid profiles of rat brain and liver mitochondria: implications for mitochondrial energy-linked functions. $J$ Membrane Biol. 2008;221(1):51-60. doi:10.1007/s00232-0079086-0
27. Sastre J, Pallardó FV, Plá R, et al. Aging of the liver: age-associated mitochondrial damage in intact hepatocytes. Hepatology. 1996;24(5):1199-1205. doi:10.1002/hep.510240536

28. Wu J-L, Wu Q-P, Peng Y-P, Zhang J-M. Effects of L-malate on mitochondrial oxidoreductases in liver of aged rats. Physiolo Res. 2011;329-336. doi:10.33549/physiolres.931986

29. Zorov DB, Juhaszova M, Sollott SJ. Mitochondrial Reactive Oxygen Species (ROS) and ROS-Induced ROS Release. Physiol Rev. 2014;94(3):909-950. doi:10.1152/physrev.00026.2013

30. Colavitti R, Finkel T. Reactive oxygen species as mediators of cellular senescence. IUBMB Life. 2005;57(4-5):277-281. doi:10.1080/15216540500091890

31. Lozada-Delgado JG, Torres-Ramos CA, Ayala-Peña S. Chapter 4 - Aging, oxidative stress, mitochondrial dysfunction, and the liver. In: Preedy VR, Patel VB, editors. Aging (Second Edition). Academic Press; 2020:37-46.

32. Pérez-Carreras M, Hoyo PD, Martín MA, et al. Defective hepatic mitochondrial respiratory chain in patients with nonalcoholic steatohepatitis. Hepatology. 2003;38(4):999-1007. doi:10.1002/ hep. 1840380426

33. Tariq Z, Green CJ, Hodson L. Are oxidative stress mechanisms the common denominator in the progression from hepatic steatosis towards non-alcoholic steatohepatitis (NASH)? Liver Int. 2014;34(7):e180-e190. doi:10.1111/liv.12523

34. Polyzos SA, Kountouras J, Mantzoros CS. Adipose tissue, obesity and non-alcoholic fatty liver disease. Minerva Endocrinol. 2017;42(2):92-108.

35. Vos B, Moreno C, Nagy N, et al. Lean non-alcoholic fatty liver disease (Lean-NAFLD): a major cause of cryptogenic liver disease. Acta Gastroenterol Belg. 2011;74(3):389-394.

36. Eguchi Y, Hyogo H, Ono M, et al. Prevalence and associated metabolic factors of nonalcoholic fatty liver disease in the general population from 2009 to 2010 in Japan: a multicenter large retrospective study. $J$ Gastroenterol. 2012;47(5):586-595. doi:10.1007/s00535-012-0533-z

37. Golabi P, Paik J, Reddy R, Bugianesi E, Trimble G, Younossi ZM. Prevalence and long-term outcomes of non-alcoholic fatty liver disease among elderly individuals from the United States. BMC Gastroenterol. 2019;19(1):56. doi:10.1186/s12876-019-0972-6

38. Younossi ZM, Koenig AB, Abdelatif D, Fazel Y, Henry L, Wymer M. Global epidemiology of nonalcoholic fatty liver disease-Meta-analytic assessment of prevalence, incidence, and outcomes. Hepatology. 2016;64(1):73-84. doi:10.1002/hep.28431

39. Singh S, Allen AM, Wang Z, Prokop LJ, Murad MH, Loomba R. Fibrosis Progression in Nonalcoholic Fatty Liver vs Nonalcoholic Steatohepatitis: a systematic review and meta-analysis of paired-biopsy studies. Clin Gastroenterol Hepatol. 2015;13 (4):643-654.e649. doi:10.1016/j.cgh.2014.04.014

40. Loomba R, Wong R, Fraysse J, et al. Nonalcoholic fatty liver disease progression rates to cirrhosis and progression of cirrhosis to decompensation and mortality: a real world analysis of Medicare data. Aliment Pharmacol Ther. 2020;51 (11):1149-1159. doi:10.1111/apt.15679

41. Dulai PS, Singh S, Patel J, et al. Increased risk of mortality by fibrosis stage in nonalcoholic fatty liver disease: systematic review and meta-analysis. Hepatology. 2017;65(5):1557-1565. doi:10.1002/hep.29085

42. Angulo P, Kleiner DE, Dam-Larsen S, et al. Liver Fibrosis, but no other histologic features, is associated with long-term outcomes of patients with nonalcoholic fatty liver disease. Gastroenterology. 2015;149(2):389-397.e310. doi:10.1053/j.gastro.2015.04.043

43. Ekstedt M, Hagström H, Nasr P, et al. Fibrosis stage is the strongest predictor for disease-specific mortality in NAFLD after up to 33 years of follow-up. Hepatology. 2015;61 (5):1547-1554. doi:10.1002/hep. 27368 
44. Younossi ZM, Stepanova M, Rafiq N, et al. Pathologic criteria for nonalcoholic steatohepatitis: interprotocol agreement and ability to predict liver-related mortality. Hepatology. 2011;53 (6):1874-1882. doi:10.1002/hep.24268

45. Kagansky N, Levy S, Keter D, et al. Non-alcoholic fatty liver disease - a common and benign finding in octogenarian patients. Liver Int. 2004;24(6):588-594.

46. Miyaaki H, Ichikawa T, Nakao K, et al. Clinicopathological study of nonalcoholic fatty liver disease in Japan: the risk factors for fibrosis. Liver Int. 2008;28(4):519-524. doi:10.1111/j.14783231.2007.01614.x

47. Lonardo A, Lombardini S, Scaglioni F, et al. Fatty liver, carotid disease and gallstones: a study of age-related associations. World $J$ Gastroenterol. 2006;12(36):5826-5833. doi:10.3748/wjg.v12. i36.5826

48. Allen AM, Therneau TM, Larson JJ, Coward A, Somers VK, Kamath PS. Nonalcoholic fatty liver disease incidence and impact on metabolic burden and death: a 20 year-community study. Hepatology. 2018;67(5):1726-1736. doi:10.1002/hep.29546

49. Labenz C, Huber Y, Kalliga E, et al. Predictors of advanced fibrosis in non-cirrhotic non-alcoholic fatty liver disease in Germany. Aliment Pharmacol Ther. 2018;48(10):1109-1116. doi:10.1111/apt.14976

50. Kaps L, Labenz C, Galle PR, Weinmann-Menke J, Kostev K, Schattenberg JM. Non-alcoholic fatty liver disease increases the risk of incident chronic kidney disease. United Eur Gastroenterol J. 2020;8(8):942-948. doi:10.1177/2050640620944098

51. Kim G-A, Lee HC, Choe J, et al. Association between non-alcoholic fatty liver disease and cancer incidence rate. J Hepatol. 2018;68(1):140-146. doi:10.1016/j.jhep.2017.09.012

52. Weinmann A, Alt Y, Koch S, et al. Treatment and survival of non-alcoholic steatohepatitis associated hepatocellular carcinoma. BMC Cancer. 2015;15:210. doi:10.1186/s12885-015-1197-x

53. Kodama K, Tokushige K, Hashimoto E, Taniai M, Shiratori K. Hepatic and extrahepatic malignancies in cirrhosis caused by nonalcoholic steatohepatitis and alcoholic liver disease. Alcohol Clin Exp Res. 2013;37(s1):E247-E252. doi:10.1111/j.15300277.2012.01900.x

54. Brunot A, Sourd SL, Pracht M, Edeline J. Hepatocellular carcinoma in elderly patients: challenges and solutions. $J$ Hepatocellular Carcinoma. 2016;3:9-18. doi:10.2147/JHC.S101448

55. Nishikawa H, Kimura $T$, Kita R, Osaki $Y$. Treatment for Hepatocellular Carcinoma in Elderly Patients: a Literature Review. J Cancer. 2013;4(8):635-643. doi:10.7150/jca.7279

56. Trevisani F, Cantarini MC, Labate AMM, et al. Surveillance for hepatocellular carcinoma in elderly Italian patients with cirrhosis: effects on cancer staging and patient survival. Am $J$ Gastroenterol. 2004;99(8):1470-1476. doi:10.1111/j.15720241.2004.30137.x

57. Marrero JA, Kulik LM, Sirlin CB, et al. Diagnosis, Staging, and Management of Hepatocellular Carcinoma: 2018 Practice Guidance by the American Association for the Study of Liver Diseases. Hepatology. 2018;68(2):723-750. doi:10.1002/ hep. 29913

58. Hung G-Y, Horng J-L, Yen H-J, Lee C-Y, Lin L-Y. Changing incidence patterns of hepatocellular carcinoma among age groups in Taiwan. J Hepatol. 2015;63(6):1390-1396. doi:10.1016/j. jhep.2015.07.032

59. White DL, Thrift AP, Kanwal F, Davila J, El-Serag HB. Incidence of Hepatocellular Carcinoma in All 50 United States, From 2000 Through 2012. Gastroenterology. 2017;152(4):812-820.e815. doi:10.1053/j.gastro.2016.11.020

60. Singh AK, Kumar R, Pandey AK. Hepatocellular Carcinoma: causes, Mechanism of Progression and Biomarkers. Curr Chem Genomics Translational Med. 2018;12:1. doi:10.2174/ 2213988501812010009
61. Younossi ZM, Golabi P, de Avila L, et al. The global epidemiology of NAFLD and NASH in patients with type 2 diabetes: a systematic review and meta-analysis. J Hepatol. 2019;71 (4):793-801. doi:10.1016/j.jhep.2019.06.021

62. Mantovani A, Byrne CD, Bonora E, Targher G. Nonalcoholic Fatty Liver Disease and Risk of Incident Type 2 Diabetes: a Meta-analysis. Diabetes Care. 2018;41(2):372-382. doi:10.2337/dc17-1902

63. Mantovani A, Petracca G, Beatrice G. et al. Non-alcoholic fatty liver disease and risk of incident chronic kidney disease: an updated meta-analysis. Gut;2020. gutjnl-2020-323082. doi:10.1136/gutjnl-2020-323082

64. Li Y, Wang J, Tang Y, et al. Bidirectional association between nonalcoholic fatty liver disease and type 2 diabetes in Chinese population: evidence from the Dongfeng-Tongji cohort study. PLoS One. 2017;12 (3):e0174291. doi:10.1371/journal.pone.0174291

65. Ekstedt M, Franzén LE, Mathiesen UL, et al. Long-term follow-up of patients with NAFLD and elevated liver enzymes. Hepatology. 2006;44(4):865-873. doi:10.1002/hep.21327

66. Francque SM, Graaff DVD, Kwanten WJ. Non-alcoholic fatty liver disease and cardiovascular risk: pathophysiological mechanisms and implications. $J$ Hepatol. 2016;65(2):425-443. doi:10.1016/j.jhep.2016.04.005

67. Hamaguchi M, Kojima T, Takeda N, et al. Nonalcoholic fatty liver disease is a novel predictor of cardiovascular disease. World $J$ Gastroenterol. 2007;13(10):1579-1584. doi:10.3748/wjg.v13. i10.1579

68. Kim D, Kim WR, Kim HJ, Therneau TM. Association between noninvasive fibrosis markers and mortality among adults with nonalcoholic fatty liver disease in the United States. Hepatology. 2013;57(4):1357-1365. doi:10.1002/hep.26156

69. Park JG, Jung J, Verma KK, et al. Liver stiffness by magnetic resonance elastography is associated with increased risk of cardiovascular disease in patients with non-alcoholic fatty liver disease. Aliment Pharmacol Ther. 2021;53(9):1030-1037.

70. Stepanova M, Younossi ZM. Independent Association Between Nonalcoholic Fatty Liver Disease and Cardiovascular Disease in the US Population. Clin Gastroenterol Hepatol. 2012;10 (6):646-650. doi:10.1016/j.cgh.2011.12.039

71. Lazo M, Hernaez R, Bonekamp S, et al. Non-alcoholic fatty liver disease and mortality among US adults: prospective cohort study. BMJ. 2011;343:d6891. doi:10.1136/bmj.d6891

72. Anstee QM, Mantovani A, Tilg H, Targher G. Risk of cardiomyopathy and cardiac arrhythmias in patients with nonalcoholic fatty liver disease. Nat Rev Gastroenterol Hepatol. 2018;15 (7):425-439. doi:10.1038/s41575-018-0010-0

73. Hattori M, Taylor TD. The human intestinal microbiome: a new frontier of human biology. DNA Res. 2009;16(1):1-12. doi:10.1093/dnares/dsn033

74. Turnbaugh PJ, Ley RE, Hamady M, Fraser-Liggett CM, Knight R, Gordon JI. The human microbiome project. Nature. 2007;449(7164):804-810. doi:10.1038/nature06244

75. Biagi E, Candela M, Fairweather-Tait S, Franceschi C, Brigidi P. Aging of the human metaorganism: the microbial counterpart. Age. 2012;34(1):247-267. doi:10.1007/s11357-011-9217-5

76. Biagi E, Nylund L, Candela M, et al. Through ageing, and beyond: gut microbiota and inflammatory status in seniors and centenarians. PLoS One. 2010;5(5):e10667. doi:10.1371/journal. pone. 0010667

77. Claesson MJ, Jeffery IB, Conde S, et al. Gut microbiota composition correlates with diet and health in the elderly. Nature. 2012;488(7410):178-184. doi:10.1038/nature11319

78. Boyce JM, Shone GR. Effects of ageing on smell and taste. Postgrad Med J. 2006;82(966):239-241. doi:10.1136/pgmj.2005.039453

79. Laurin D, Brodeur JM, Bourdages J, Vallée R, Lachapelle D. Fibre intake in elderly individuals with poor masticatory performance. J Can Dent Assoc. 1994;60(5):443-446. 
80. Oudshoorn C, van der Cammen TJ, McMurdo ME, van Leeuwen JP, Colin EM. Ageing and vitamin D deficiency: effects on calcium homeostasis and considerations for vitamin D supplementation. $\quad B r \quad J \quad$ Nutr. 2009;101(11):1597-1606. doi:10.1017/S0007114509338842

81. Maynard CL, Weaver CT. Intestinal effector T cells in health and disease. Immunity. 2009;31(3):389-400. doi:10.1016/j. immuni.2009.08.012

82. Larbi A, Franceschi C, Mazzatti D, Solana R, Wikby A, Pawelec G. Aging of the immune system as a prognostic factor for human longevity. Physiology. 2008;23:64-74.

83. Schneider KM, Bieghs V, Heymann F, et al. CX3CR1 is a gatekeeper for intestinal barrier integrity in mice: limiting steatohepatitis by maintaining intestinal homeostasis. Hepatology. 2015;62(5):1405-1416. doi:10.1002/hep.27982

84. Bertolotti M, Lonardo A, Mussi C, et al. Nonalcoholic fatty liver disease and aging: epidemiology to management. World $J$ Gastroenterol. 2014;20(39):14185-14204. doi:10.3748/wjg.v20. i39.14185

85. Caussy C, Tripathi A, Humphrey G, et al. A gut microbiome signature for cirrhosis due to nonalcoholic fatty liver disease. Nat Commun. 2019;10(1):1406. doi:10.1038/s41467-019-09455-9

86. Koliada A, Syzenko G, Moseiko V, et al. Association between body mass index and Firmicutes/Bacteroidetes ratio in an adult Ukrainian population. BMC Microbiol. 2017;17(1):120. doi:10.1186/s12866-017-1027-1

87. Mueller S, Saunier K, Hanisch C, et al. Differences in fecal microbiota in different European study populations in relation to age, gender, and country: a cross-sectional study. Appl Environ Microbiol. 2006;72 (2):1027-1033. doi:10.1128/AEM.72.2.1027-1033.2006

88. Janssen I, Heymsfield SB, Ross R. Low relative skeletal muscle mass (sarcopenia) in older persons is associated with functional impairment and physical disability. J Am Geriatr Soc. 2002;50 (5):889-896. doi:10.1046/j.1532-5415.2002.50216.x

89. Tilg $\mathrm{H}$, Moschen AR. Insulin resistance, inflammation, and non-alcoholic fatty liver disease. Trends Endocrinol Metab. 2008;19(10):371-379. doi:10.1016/j.tem.2008.08.005

90. Abbatecola AM, Paolisso G, Fattoretti P, et al. Discovering pathways of sarcopenia in older adults: a role for insulin resistance on mitochondria dysfunction. J Nutr Health Aging. 2011;15 (10):890-895. doi:10.1007/s12603-011-0366-0

91. Hong HC, Hwang SY, Choi HY, et al. Relationship between sarcopenia and nonalcoholic fatty liver disease: the Korean Sarcopenic Obesity Study. Hepatology. 2014;59(5):1772-1778. doi:10.1002/hep. 26716

92. Beyer I, Mets T, Bautmans I. Chronic low-grade inflammation and age-related sarcopenia. Curr Opin Clin Nutr Metab Care. 2012;15(1):12-22. doi:10.1097/MCO.0b013e32834dd297

93. Electrophysiology TFOTESOCTNA. Heart rate variability: standards of measurement, physiological interpretation and clinical use. Task Force of the European Society of Cardiology and the North American Society of Pacing and Electrophysiology. Circulation. 1996;93 (5):1043-1065. doi:10.1161/01.CIR.93.5.1043

94. Liu YC, Hung CS, Wu YW, et al. Influence of non-alcoholic fatty liver disease on autonomic changes evaluated by the time domain, frequency domain, and symbolic dynamics of heart rate variability. PLoS One. 2013;8(4):e61803. doi:10.1371/journal.pone.0061803

95. Newton JL. Systemic symptoms in non-alcoholic fatty liver disease. Dig Dis. 2010;28(1):214-219. doi:10.1159/000282089

96. Frith J, Newton JL. Autonomic dysfunction in chronic liver disease. Liver Int. 2009;29(4):483-489. doi:10.1111/j.14783231.2009.01985.x

97. Newton JL, Hollingsworth KG, Taylor R, et al. Cognitive impairment in primary biliary cirrhosis: symptom impact and potential etiology. Hepatology. 2008;48(2):541-549. doi:10.1002/hep.22371
98. Forton DM, Thomas HC, Murphy CA, et al. Hepatitis C and cognitive impairment in a cohort of patients with mild liver disease. Hepatology. 2002;35(2):433-439. doi:10.1053/ jhep.2002.30688

99. Yilmaz Y, Ozdogan O. Liver disease as a risk factor for cognitive decline and dementia: an under-recognized issue. Hepatology. 2009;49(2):698. doi:10.1002/hep.22752

100. Brea A, Mosquera D, Martín E, Arizti A, Cordero JL, Ros E. Nonalcoholic fatty liver disease is associated with carotid atherosclerosis: a case-control study. Arterioscler Thromb Vasc Biol. 2005;25(5):1045-1050. ATV.0000160613.57985.18

101. Abbatecola AM, Ferrucci L, Marfella R, Paolisso G. Insulin resistance and cognitive decline may be common soil for frailty syndrome. Arch Intern Med. 2007;167(19):2145-2146. doi:10.1001/archinte.167.19.2145-b

102. Koplay M, Gulcan E, Ozkan F. Association between serum vitamin B12 levels and the degree of steatosis in patients with nonalcoholic fatty liver disease. $J$ Investig Med. 2011;59 (7):1137-1140. doi:10.2310/JIM.0b013e31822a29f5

103. Sepe A, Tchkonia T, Thomou T, Zamboni M, Kirkland JL. Aging and regional differences in fat cell progenitors - a mini-review. Gerontology. 2011;57(1):66-75. doi:10.1159/000279755

104. Tchkonia T, Morbeck DE, Von Zglinicki T, et al. Fat tissue, aging, and cellular senescence. Aging Cell. 2010;9(5):667-684. doi:10.1111/j.1474-9726.2010.00608.x

105. Spalding KL, Arner E, Westermark PO, et al. Dynamics of fat cell turnover in humans. Nature. 2008;453(7196):783-787. doi:10.1038/nature06902

106. Bays HE, González-Campoy JM, Bray GA, et al. Pathogenic potential of adipose tissue and metabolic consequences of adipocyte hypertrophy and increased visceral adiposity. Expert Rev Cardiovasc Ther. 2008;6(3):343-368.

107. Guo W, Pirtskhalava T, Tchkonia T, et al. Aging results in paradoxical susceptibility of fat cell progenitors to lipotoxicity. Am $J$ Physiol Endocrinol Metab. 2007;292(4):E1041-1051. doi:10.1152/ajpendo.00557.2006

108. Suganami T, Tanimoto-Koyama K, Nishida J, et al. Role of the Toll-like receptor 4/NF-kappaB pathway in saturated fatty acid-induced inflammatory changes in the interaction between adipocytes and macrophages. Arterioscler Thromb Vasc Biol. 2007;27(1):84-91. doi:10.1161/01.ATV.0000251608.09329.9a

109. Finelli C, Sommella L, Gioia S, La Sala N, Tarantino G. Should visceral fat be reduced to increase longevity? Ageing Res Rev. 2013;12(4):996-1004. doi:10.1016/j.arr.2013.05.007

110. Cheng H-Y, Wang H-Y, Chang W-H, et al. Nonalcoholic Fatty Liver Disease: prevalence, Influence on Age and Sex, and Relationship with Metabolic Syndrome and Insulin Resistance. Int $J \quad$ Gerontol. 2013;7(4):194-198. doi:10.1016/j. ijge.2013.03.008

111. American Diabetes Association. Comprehensive Medical Evaluation and Assessment of Comorbidities: standards of Medical Care in Diabetes-2020. Diabetes Care. 2020;43 (Supp11):S37-S47. doi:10.2337/dc20-S004

112. Browning JD, Szczepaniak LS, Dobbins R, et al. Prevalence of hepatic steatosis in an urban population in the United States: impact of ethnicity. Hepatology. 2004;40(6):1387-1395. doi: $10.1002 /$ hep. 20466

113. Sorrentino P, Tarantino G, Conca P, et al. Silent non-alcoholic fatty liver disease-a clinical-histological study. $J$ Hepatol. 2004;41(5):751-757. doi:10.1016/j.jhep.2004.07.010

114. Fracanzani AL, Valenti L, Bugianesi E, et al. Risk of severe liver disease in nonalcoholic fatty liver disease with normal aminotransferase levels: a role for insulin resistance and diabetes. Hepatology. 2008;48(3):792-798. doi:10.1002/hep.22429 
115. Kotronen A, Peltonen M, Hakkarainen A, et al. Prediction of Non-Alcoholic Fatty Liver Disease and Liver Fat Using Metabolic and Genetic Factors. Gastroenterology. 2009;137 (3):865-872. doi:10.1053/j.gastro.2009.06.005

116. Huang $\mathrm{X}, \mathrm{Xu} \mathrm{M}$, Chen $\mathrm{Y}$, et al. Validation of the Fatty Liver Index for Nonalcoholic Fatty Liver Disease in Middle-Aged and Elderly Chinese. Medicine. 2015;94(40):e1682. doi:10.1097/ MD.0000000000001682

117. Sviklāne L, Olmane E, Dzērve Z, Kupčs K, Pīrāgs V, Sokolovska J. Fatty liver index and hepatic steatosis index for prediction of non-alcoholic fatty liver disease in type 1 diabetes. J Gastroenterol Hepatol. 2018;33(1):270-276. doi:10.1111/ jgh. 13814

118. Byrne CD. EASL-EASD-EASO Clinical Practice Guidelines for the management of non-alcoholic fatty liver disease. $J$ Hepatol. 2016;64(6):1388-1402. doi:10.1016/j.jhep.2015.11.004

119. Adams LA, George J, Bugianesi E, et al. Complex non-invasive fibrosis models are more accurate than simple models in non-alcoholic fatty liver disease. $J$ Gastroenterol Hepatol. 2011;26(10):1536-1543. doi:10.1111/j.1440-1746.2011.06774.x

120. Angulo P, Hui JM, Marchesini G, et al. The NAFLD fibrosis score: a noninvasive system that identifies liver fibrosis in patients with NAFLD. Hepatology. 2007;45(4):846-854. doi:10.1002/ hep. 21496

121. McPherson S, Hardy T, Dufour J-F, et al. Age as a Confounding Factor for the Accurate Non-Invasive Diagnosis of Advanced NAFLD Fibrosis. Official j Am College Gastroenterol. 2017;112 (5):740-751. doi:10.1038/ajg.2016.453

122. Pitisuttithum P, Chan WK, Piyachaturawat $P$, et al. Predictors of advanced fibrosis in elderly patients with biopsy-confirmed nonalcoholic fatty liver disease: the GOASIA study. $B M C$ Gastroenterol. 2020;20(1):88. doi:10.1186/s12876-020-01240-z

123. Michel M, Schattenberg JM. Liver-specific diagnostic for non-alcoholic fatty liver disease (NAFLD) - time to replace liver biopsy?. Z Gastroenterol. 2020;58(12):1233-1240.

124. Castera L, Friedrich-Rust M, Loomba R. Noninvasive Assessment of Liver Disease in Patients With Nonalcoholic Fatty Liver Disease. Gastroenterology. 2019;156(5):1264-1281. e1264. doi:10.1053/j.gastro.2018.12.036

125. Imajo K, Kessoku $\mathrm{T}$, Honda $\mathrm{Y}$, et al. Magnetic Resonance Imaging More Accurately Classifies Steatosis and Fibrosis in Patients With Nonalcoholic Fatty Liver Disease Than Transient Elastography. Gastroenterology. 2016;150(3):626-637.e627. doi:10.1053/j.gastro.2015.11.048

126. Wong VW-S, Vergniol J, Wong GL-H, et al. Diagnosis of fibrosis and cirrhosis using liver stiffness measurement in nonalcoholic fatty liver disease. Hepatology. 2010;51(2):454-462. doi:10.1002/ hep. 23312

127. Gilmore IT, Burroughs A, Murray-Lyon IM, Williams R, Jenkins D, Hopkins A. Indications, methods, and outcomes of percutaneous liver biopsy in England and Wales: an audit by the British Society of Gastroenterology and the Royal College of Physicians of London. Gut. 1995;36(3):437-441. doi:10.1136/ gut.36.3.437

128. Eslam M, Sanyal AJ, George J. MAFLD: a Consensus-Driven Proposed Nomenclature for Metabolic Associated Fatty Liver Disease. Gastroenterology. 2020;158(7):1999-2014.e1991. doi:10.1053/j.gastro.2019.11.312

129. Fouad Y, Waked I, Bollipo S, Gomaa A, Ajlouni Y, Attia D. What's in a name? Renaming 'NAFLD' to 'MAFLD'. Liver Int. 2020;40(6):1254-1261. doi:10.1111/liv.14478

130. Promrat K, Kleiner DE, Niemeier HM, et al. Randomized controlled trial testing the effects of weight loss on nonalcoholic steatohepatitis. Hepatology. 2010;51(1):121-129. doi:10.1002/ hep. 23276
131. Vilar-Gomez E, Martinez-Perez Y, Calzadilla-Bertot L, et al. Weight Loss Through Lifestyle Modification Significantly Reduces Features of Nonalcoholic Steatohepatitis. Gastroenterology. 2015;149(2):367-378.e365. doi:10.1053/j. gastro.2015.04.005

132. Wt A, Wv L, Mc H, Bb A. Lifestyle Modification for Obesity. Circulation. 2012;125(9):1157-1170. doi:10.1161/CIRCULATIO NAHA.111.039453

133. Aggarwal B, Liao M, Allegrante JP, Mosca L. Low Social Support Level is Associated with Non-Adherence to Diet at 1 Year in the Family Intervention Trial for Heart Health (FIT Heart). J Nutr Educ Behav. 2010;42(6):380-388. doi:10.1016/j. jneb.2009.08.006

134. Krukowski RA, Harvey-Berino J, Bursac Z, Ashikaga T, West DS. Patterns of success: online self-monitoring in a web-based behavioral weight control program. Health Psychol. 2013;32(2):164-170. doi:10.1037/a0028135

135. Ryu S, Chang Y, Jung H-S, et al. Relationship of sitting time and physical activity with non-alcoholic fatty liver disease. J Hepatol. 2015;63(5):1229-1237. doi:10.1016/j.jhep.2015.07.010

136. Romero-Gómez M, Zelber-Sagi S, Trenell M. Treatment of NAFLD with diet, physical activity and exercise. J Hepatol. 2017;67(4):829-846. doi:10.1016/j.jhep.2017.05.016

137. Evans C. Malnutrition in the elderly: a multifactorial failure to thrive. Perm J. 2005;9(3):38-41. doi:10.7812/TPP/05-056

138. Sieber CC. Malnutrition and sarcopenia. Aging Clin Exp Res. 2019;31(6):793-798. doi:10.1007/s40520-019-01170-1

139. Yang X, Xu Z, Zhang C, Cai Z, Zhang J. Metformin, beyond an insulin sensitizer, targeting heart and pancreatic $\beta$ cells. Biochimica et Biophysica Acta. 2017;1863(8):1984-1990. doi:10.1016/j.bbadis.2016.09.019

140. Li Y, Liu L, Wang B, Wang J, Chen D. Metformin in non-alcoholic fatty liver disease: a systematic review and meta-analysis. Biomed Rep. 2013;1(1):57-64. doi:10.3892/ br. 2012.18

141. Rizos CV, Kei A, Elisaf MS. The current role of thiazolidinediones in diabetes management. Arch Toxicol. 2016;90 (8):1861-1881.

142. Bailey CJ. Treating insulin resistance in type 2 diabetes with metformin and thiazolidinediones. Diabetes Obes Metab. 2005;7 (6):675-691. doi:10.1111/j.1463-1326.2005.00497.x

143. Musso G, Cassader M, Paschetta E, Gambino R. Thiazolidinediones and Advanced Liver Fibrosis in Nonalcoholic Steatohepatitis: a Meta-analysis. JAMA Intern Med. 2017;177(5):633. doi:10.1001/jamainternmed.2016.9607

144. Bril F, Kalavalapalli S, Clark VC, et al. Response to Pioglitazone in Patients With Nonalcoholic Steatohepatitis With vs Without Type 2 Diabetes. Clin Gastroenterol Hepatol. 2018;16(4):558566.e552. doi:10.1016/j.cgh.2017.12.001

145. Belfort R, Harrison SA, Brown K, et al. A Placebo-Controlled Trial of Pioglitazone in Subjects with Nonalcoholic Steatohepatitis. $N$ Eng J Med. 2006;355(22):2297-2307. doi:10.1056/NEJMoa060326

146. Sanyal AJ, Chalasani N, Kowdley KV, et al. Pioglitazone, Vitamin E, or Placebo for Nonalcoholic Steatohepatitis. N Eng J Med. 2010;362(18):1675-1685. doi:10.1056/NEJMoa0907929

147. Hernandez AV, Usmani A, Rajamanickam A, Moheet A. Thiazolidinediones and Risk of Heart Failure in Patients with or at High Risk of Type 2 Diabetes Mellitus. Am J Cardiovascular Drugs. 2011;11(2):115-128. doi:10.2165/11587580-00000000000000

148. Kaku K, Hashiramoto M. Thiazolidinediones and bone fractures. $J$ Diabetes Investig. 2011;2(5):354-355. doi:10.1111/j.20401124.2011.00142.x

149. Ensrud KE. Epidemiology of Fracture Risk With Advancing Age. J Gerontolo. 2013;68(10):1236-1242. doi:10.1093/gerona/glt092 
150. Lu Y, Hajifathalian K, Ezzati M, Woodward M, Rimm EB, Danaei G. Metabolic mediators of the effects of body-mass index, overweight, and obesity on coronary heart disease and stroke: a pooled analysis of 97 prospective cohorts with 1.8 million participants. Lancet. 2014;383(9921):970-983.

151. Wang XX, Jiang $T$, Shen $Y$, et al. The farnesoid $X$ receptor modulates renal lipid metabolism and diet-induced renal inflammation, fibrosis, and proteinuria. Am J Physiol Renal Physiol. 2009;297(6):F1587-F1596. doi:10.1152/ajprenal.00404.2009

152. Mudaliar S, Henry RR, Sanyal AJ, et al. Efficacy and Safety of the Farnesoid X Receptor Agonist Obeticholic Acid in Patients With Type 2 Diabetes and Nonalcoholic Fatty Liver Disease. Gastroenterology. 2013;145(3):574-582.e571. doi:10.1053/j. gastro.2013.05.042

153. Neuschwander-Tetri BA, Loomba R, Sanyal AJ, et al. Farnesoid $\mathrm{X}$ nuclear receptor ligand obeticholic acid for non-cirrhotic, non-alcoholic steatohepatitis (FLINT): a multicentre, randomised, placebo-controlled trial. Lancet. 2015;385(9972):956-965. doi:10.1016/S0140-6736(14)61933-4

154. Younossi ZM, Ratziu V, Loomba R, et al. Obeticholic acid for the treatment of non-alcoholic steatohepatitis: interim analysis from a multicentre, randomised, placebo-controlled phase 3 trial. Lancet. 2019;394(10215):2184-2196. doi:10.1016/S01406736(19)33041-7

155. Katout M, Zhu H, Rutsky J, et al. Effect of GLP-1 Mimetics on Blood Pressure and Relationship to Weight Loss and Glycemia Lowering: results of a Systematic Meta-Analysis and Meta-Regression. Am J Hypertens. 2014;27(1):130-139. doi:10.1093/ajh/hpt196

156. Sun F, Wu S, Wang J, et al. Effect of Glucagon-like Peptide-1 Receptor Agonists on Lipid Profiles Among Type 2 Diabetes: a Systematic Review and Network Meta-analysis. Clin Ther. 2015;37(1):225-241.e228. doi:10.1016/j.clinthera.2014.11.008
157. Armstrong MJ, Gaunt P, Aithal GP, et al. Liraglutide safety and efficacy in patients with non-alcoholic steatohepatitis (LEAN): a multicentre, double-blind, randomised, placebo-controlled phase 2 study. Lancet. 2016;387(10019):679-690. doi:10.1016/ S0140-6736(15)00803-X

158. Newsome PN, Buchholtz K, Cusi K, et al. A Placebo-Controlled Trial of Subcutaneous Semaglutide in Nonalcoholic Steatohepatitis. $N$ Eng J Med. 2020;2:4.

159. Staiger H, Keuper M, Berti L. Hrabě de Angelis M, Häring H-U. Fibroblast Growth Factor 21-Metabolic Role in Mice and Men. Endocr Rev. 2017;38(5):468-488.

160. Verzijl CRC, Peppel IPVD, Struik D, Jonker JW. Pegbelfermin (BMS-986036): an investigational PEGylated fibroblast growth factor 21 analogue for the treatment of nonalcoholic steatohepatitis. Expert Opin Investig Drugs. 2020;29(2):125-133.

161. Squibb B-M. A Phase 2B Randomized, Double-Blind, PlaceboControlled Study Evaluating the Safety and Efficacy of BMS-986036 (PEG-FGF21) in Adults with Nonalcoholic Steatohepatitis (NASH) and Compensated Liver Cirrhosis. clinicaltrials.gov; 2020.

162. Squibb B-M. A Phase 2B Randomized Double-Blind, PlaceboControlled Study Evaluating the Safety and Efficacy of BMS-986036 (PEG-FGF21) in Adults with Nonalcoholic Steatohepatitis (NASH) and Stage 3 Liver Fibrosis. clinicaltrials.gov; 2021.

163. Emerging YZ. Research on MGL-3196 for the Treatment of Nonalcoholic Steatohepatitis. Gastroenterol Hepatol ( $N$ Y). 2019;15(6):317-319.

164. Madrigal Pharmaceuticals I. A Phase 3, Multinational, DoubleBlind, Randomized, Placebo-Controlled Study of MGL-3196 (Resmetirom) in Patients with Non-Alcoholic Steatohepatitis (NASH) and Fibrosis to Resolve NASH and Reduce Progression to Cirrhosis And/Or Hepatic Decompensation. clinicaltrials.gov; 2021 .
Clinical Interventions in Aging

\section{Publish your work in this journal}

Clinical Interventions in Aging is an international, peer-reviewed journal focusing on evidence-based reports on the value or lack thereof of treatments intended to prevent or delay the onset of maladaptive correlates of aging in human beings. This journal is indexed on PubMed Central, MedLine, CAS, Scopus and the Elsevier
Bibliographic databases. The manuscript management system is completely online and includes a very quick and fair peer-review system, which is all easy to use. Visit http://www.dovepress.com/ testimonials.php to read real quotes from published authors. 